\title{
Analysis of the Usage of Magnetic Force-directed Approach and Visual Techniques for Interactive Context-based Drawing of Multi-attributed Graphs
}

\author{
Vitaly Zabiniako ${ }^{1}$, Pavel Rusakov ${ }^{2}$, \\ ${ }^{1}$ ABC Software, Latvia, ${ }^{2}$ Riga Technical University, Latvia
}

\begin{abstract}
In this article, the authors perform an analysis in order to assess adaptation of magnetic force-directed algorithms for context-based information extraction from multi-attributed graphs during visualization sessions. Theoretic standings behind magnetic force-directed approach are stated together with review on how particular features of respective algorithms in combination with appropriate visual techniques are especially suitable for improved processing and presenting of knowledge that is captured in form of graphs. The complexity of retrieving multi-attributed information within the proposed approach is handled with dedicated tools, such as selective attraction of nodes to MFE (Magnetic Force Emitter) based on search criteria, localization of POI (Point of Interest) regions, graph node anchoring, etc. Implicit compatibility of aforementioned tools with interactive nature of data exploration is distinguished. Description of case study, based on bibliometric network analysis is given, which is followed by the review of existing related works in this field. Conclusions are made and further studies in the field of visualization of multi-attributed graphs are defined.
\end{abstract}

Keywords - Graph, context-based, force-directed, visualization.

\section{INTRODUCTION}

It is a well-known fact that nowadays possession of relevant information and its effective processing are the key points for achieving success in business, scientific research, education or any other domain of human activity. Considering that the amount of data we have to deal with in everyday scenarios has drastically increased during the past few decades, it is of great importance for the end user to be able to identify and extract exactly those data subsets which are of interest in scope of his/her current tasks. Information visualization software is one of the powerful tools (along with knowledge-based systems, computer-aided design applications, model-driven engineering and other modern approaches) that brings the ability not only to represent related information conveniently to the user, but also to interact with it, allowing for searching, filtering, selecting and reasoning activities in a dynamic, goal-based manner.

Another aspect is the multi-attribute nature of information that inevitably arises in data sets with hundreds and thousands of entities. The simplest example of this is the bibliographic problem domain - even while given the access to an electronic library catalogue, one has to traverse plethora of records to identify and retrieve those works relevant to the topic being searched. This process usually deals with exploration of different authors, annotations, tables of contents, keywords, references etc. This calls for an improved computer-based solution that enhances traditional taxonomical, anthological and classification-based approaches.

We see a great potential in the involvement of interactive visualization for the mentioned and similar tasks by capturing all available information in formalized form of attributed graphs and applying a force-directed graph drawing approach (to be more specific - its magnetic subclass) together with a set of appropriate visual techniques for aiding aforementioned data processing tasks. We argue that implicit features of the concept of magnetic forces and mutual interaction of those are coherent with many common interaction scenarios between the user and data entities and can be used to benefit successful solution of such tasks.

Given this, the goal of this research is to support further development of tools and methods that involve the usage of an interactive magnetic force-directed approach in information visualization for effective processing of information within multi-attribute datasets.

To reach this goal, the following six tasks are defined: 1) to provide general overview and theory behind magnetic forcedirected information visualization; 2) to define suitable visualization approaches and tools for improved data manipulation that are based on the aforementioned concept; 3 ) to propose modifications of canonic force-directed drawing algorithms which allow for the implementation of appropriate changes; 4) to perform a case study in order to assess usability of the method in the field of bibliometric network analysis; 5) to review existing related works in this field; 6) to make a conclusion about achieved results and outline potential further work.

In Section II of this paper, common principles of magnetic force-directed graph drawing are outlined. Section III describes magnetic force emitters, local regions of interest, anchoring of graph nodes, visual techniques and other suitable means for context-based interaction with data. Section IV defines theoretic standings for proposed changes and calculations of appropriate force coefficients. Sections V and VI respectively present a case study and description of the current state of the art in this field. Section VII provides summary of the present research and highlights key aspects of its potential future development. 


\section{MAGNETIC FORCE-DIRECTED GRAPH DRAWING}

Force-directed layout is among the most popular approaches to drawing graphs nowadays. The original description of this method was proposed by P. Eades in 1984 [1]. The concept of electromechanical system was used by representing nodes with steel rings, replacing edges with springs and allowing for the system to move into a balanced state that is characterized by minimum kinetic energy.

Two types of forces between nodes exist in this model - the force of attraction $F_{a}$ which occurs only between adjacent nodes and the force of repulsion $F_{r}$ which is calculated for all nodes. The exact calculation of repulsion and attraction forces is given as in (1):

$$
F_{a}=c_{1} \log \left(\frac{d}{c_{2}}\right) ; F_{r}=\frac{c_{3}}{\sqrt{d}},
$$

where $d$-a geometrical distance between two nodes; $c_{1}, c_{2}$, $c_{3}$ - parameters for tuning the model.

Considering that a constant number of iterations $M$ cannot guarantee the quality of the resulting layout for any arbitrary graph, another approach to conditional stop is introduced which is based on comparing a threshold value with average energy of the system that is dynamically calculated according to (2):

$$
\bar{E}=\frac{1}{n} \sum_{i=1}^{n}\left|F_{a, i}+F_{r, i}\right|,
$$

where $\bar{E}$ - average kinetic energy; $n$-a number of nodes. Initial placement of vertices in random positions and further iterative refinements yield the pattern that is common for all force-directed approaches as in Fig. 1.

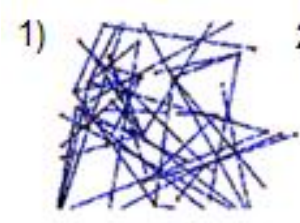

$\mathrm{M}=0$

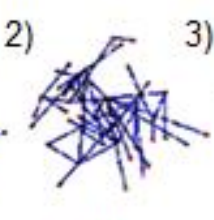

$M=10$
3)

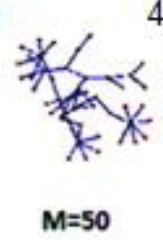

4)

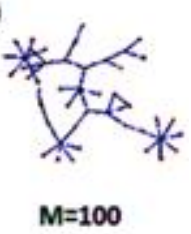

Fig. 1 Emerging of force-directed graph pattern.

An additional type of force was proposed by K. Sugiyama in [2]. This work introduces the concept of magnetic-spring graph model that is placed in different types of magnetic fields. This model states that nodes connected by a spring can be affected either by attractive spring force $F_{a s}$ or repulsive spring force $F_{r s}$, unconnected nodes produce mutual repulsive force $F_{r}$ and magnetic force $F_{m}$ is simulated for all graph edges.

The calculation of $F_{m}$ is given in (3):

$$
F_{m}=c_{m} b d^{\alpha} \theta^{\beta},
$$

where $d$ - a distance between connected nodes; $\theta$ - an angle from orientation of magnetic field to orientation of edge $(-\pi<\theta<\pi) ; c_{m}, b, \alpha, \beta$ - parameters for tuning the model.

Three main and two compound types of magnetic force field are defined:

- parallel field that is represented by a constant force vector $(0,1)$ in a two-dimensional space for north; $(-1,0)$ west; $(0,-1)$ south; $(1,0)$ for east orientation of the field see Fig. 2, part 1;

- polar field which is defined as $\frac{(x, y)}{|(x, y)|}-$ see Fig. 2, part 2;

- concentric field which is defined as $\frac{(y,-x)}{|(x, y)|}$ for clockwise and as $\frac{(-y, x)}{|(x, y)|}$ for counter-clockwise - see Fig. 2, part 3;

- compound orthogonal filed (two parallel fields crossed) see Fig. 2, part 4;

- compound polar-concentric field (combination of both polar and concentric fields) - see Fig. 2, part 5.
1)

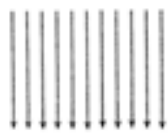

4)

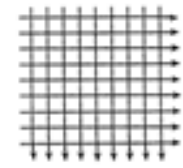

2)

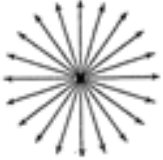

3)

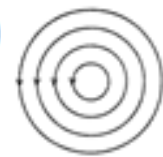

5)

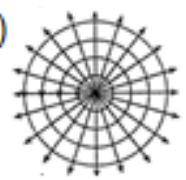

Fig. 2. Types of force fields in a magnetic-spring model.

Empiric analysis of properties of force-directed layout reveals implicit useful features of this approach, such as:

1. Ability to auto-outline geometrically localized clusters of elements with dense mutual relations, while keeping decoupled clusters separate;

2. Minimization of occupied space which results in reduced graph bounding box;

3. Auto-outlining of symmetric structures in the graph;

4. Similar implementation for visualization of graphs both in two-dimensional and three-dimensional space that is based on adding of the depth component $(x, y) \rightarrow(x, y, z)$.

5. Dynamic evolving of the resulting layout that can be presented as an interactive animation;

6. Ability to use additional force fields for altering the balanced layout emerging pattern.

These properties enable usage of magnetic force-directed approach for visualization of the graph that is based on dynamic interaction with the user. According to this approach, the mentioned constant number of iterations (or conditional stop) is removed from the algorithm that leaves the model of a graph in a permanent semi-active standby state even when a well-formed and balanced layout has emerged. This state of the model enables potential useful reactions to additional external forces, which might be introduced by the user on purpose. Description of related logic behind this process is provided in the next section. 


\section{MODELS FOR CONTEXT-BASED GRAPH DRAWING}

Before the interactive visualization itself can take place, the data being processed must be converted into an appropriate format, i.e., an attributed graph that is defined as $G=\{V, E, X\}$ where $V$ is a set of vertices (nodes) $N_{v}, E$ is a set of edges $N_{e}$ so that $E \subset V \times V$ and an edge $\left(v_{i}, v_{j}\right)$ represents a relationship between $v_{i}$ and $v_{j} . X$ is a set of $W$-dimensional feature vectors $x_{i} \in X$ that are paired with corresponding vertices $v_{i} \in V$ and represent characteristics of $v_{i}$ (for example, if $v_{i}$ represents a student, the $w$-th characteristic $x_{i}[w]$ can hold such attributes as Name, Surname, Age, etc.). The process of conversion itself, although domain-specific, is pretty straightforward and falls out of the scope of this research.

Below we will list a set of methods for context-based interaction with data.

Magnetic Force Emitters (MFEs). As it was mentioned, an external force might be applied to the model of $G$ that was in a standby state. We will define the source of such force as MFEs (refer to [3] for a general description of this concept). In its essence, MFE is a spatially localized point with precise coordinates that is characterized either by the magnitude of attraction $\left(F_{M F E+}\right)$ or repulsion $\left(F_{M F E-}\right)$ of elements of $V$. Considering that each node can hold multiple $x_{i}$ attributes, it is appropriate to introduce the same approach to MFE by defining $\{M F E, \quad X\}$ so that $x_{i} \in X$ is paired with $M F E_{i} \in M F E$ and represent characteristics of $M F E_{i}$. This allows applying different attributes to single MFE that will interact with different attributes of multiple nodes at the same time.

An example of $F_{M F E+}$ interaction would be placing and configuring the MFE in a chosen space region in order to "attract" to this particular position nodes that conforms to user-defined criteria for further inspection. This scenario is presented in Fig. 3.
1)

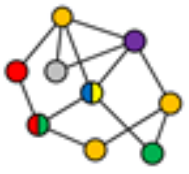

3)

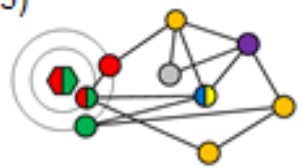

2)

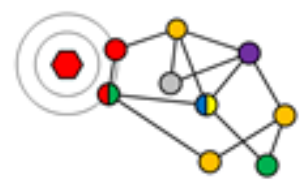

4)

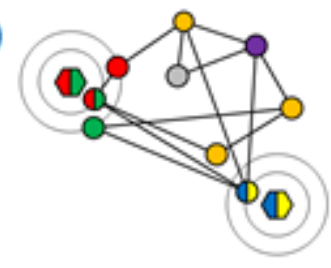

Fig. 3. Base scenarios for multi-attributed interaction of MFE.

Part 1 outlines initial configuration of graph model. Part 2 demonstrates introduction of the first MFE to a single attribute [red] that attracts nodes with the same attribute (both [red] and [red]+[green] are conformant). In this case, calculation of the force effect between MFE and arbitrary node is based on $F_{a}$ in (1). Part 3 demonstrates the case when multi-attributed MFE interacts with multiple attributes of nodes. In this case, those nodes with a greater number of conformant attributes are affected with proportionally greater force (refer to Section 4 for a calculation method). Part 4 demonstrates introduction of the second MFE that affects the graph model independently.

Contrary to the aforementioned approach, application of $F_{M F E-}$ type forces encourages placing the MFE near (or within) the graph body to "repulse" nodes that must be filtered out of the inspection based on the unwanted criteria.

Abilities to use a) multi-attributed and b) opposite directing emitters are fully orthogonal and can be used in any combination suitable for achieving user goals.

Anchoring of graph nodes. Although dynamic nature of a graph model in a standby state is of benefit for interactive manipulation, the need to geometrically fix subpart of its current topology may arise at some point. Usually this is the case when the preliminary iteration of context-based search has been performed and a number of potential candidate results have already emerged from initial placement of a graph model. To prevent losing these results, any further interaction between these particular nodes, MFE and the rest of the nodes, must be nullified for next iterations - see Fig. 4.
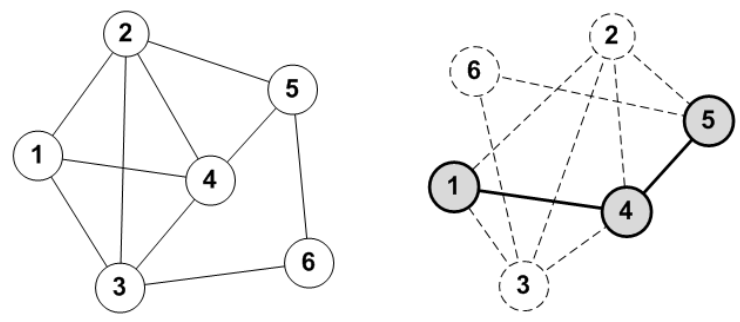

Fig. 4. Anchoring of graph nodes.

This is done by omitting calculations for updating of positions of these nodes (optionally - also by preventing other nodes from being affected by these locked elements and treating them as completely "transparent" for the rest of the graph).

Point of Interest (POI) and Region of Interest (ROI). A common visualization workflow consists of iterative introduction and adjustment of MFE and monitoring of reaction of the graph model. Considering that attracted and repulsed graph nodes may be localized in different spatial positions after interaction with MFE, it is of great importance to be able to effectively identify "points of interest" around which suitable results are grouped.

We define a POI as a geometric point which has: a) target attributes; b) density threshold value; c) coordinates $\mathrm{x}, \mathrm{y},[\mathrm{z}]$; d) magnitude. Given only the spatial model of an attributed graph, the calculation of a set of POI is as follows:

1. Define a set of target attributes and wanted density threshold (input);

2. Divide the graph model to separate sectors using the spatial grid (refer to [4]).

3. Iterate through all sectors and mark those with a number of conformant graph nodes equal or more than the density threshold; 
4. Merge marked neighbor sectors into clusters with magnitude $M$ equal to number of merged sectors;

5. Calculate centroid of each cluster from central points of corresponding sectors and add its coordinates $\{x, y,[z]\}$ and $M$ to a set of resulting POI (output).

Visual representation of respective process is presented in Fig. 5.
1)

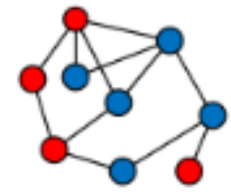

3)

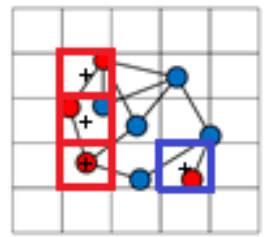

2)

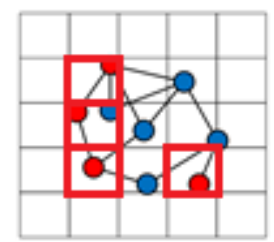

4)

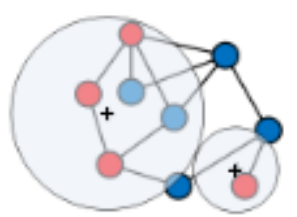

Fig. 5. Identification of POI and ROI.

The magnitude of POI allows expanding this concept into the "region of interests" (ROI) by defining a circular (spherical in 3D) subpart of the space, where POI is the center and $M$ represents a radius.

Visual techniques. Identification of POI and ROI enables the usage of additional tools for improved information comprehension. After relevant nodes are localized via MFE and anchoring, the user must concentrate on further inspection of respective regions while abstracting from the rest of the data. This can be done by using appropriate visual techniques (refer to [5] for a detailed description).

Some of these techniques are particularly useful due to direct conformance of their nature with the task being solved, e.g. ,transparency (Fig. 6, part 1), magnification (Fig. 6, part 2), distance dependent illumination (Fig. 6, part 3) and blurring (Fig. 6, part 4).
1)

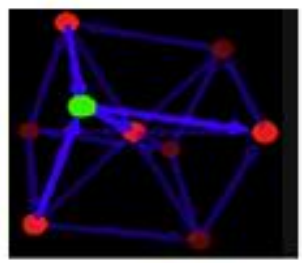

3)

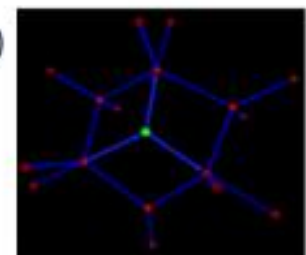

2)

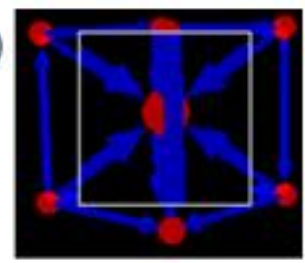

4)

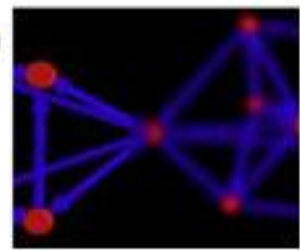

Fig. 6. Application of visual techniques for ROI.
The area of application of these techniques is defined by ROI. In its turn, POI might be used in those techniques that require a reference point (for example - as in distance dependent illumination).

Aside from the mentioned techniques, "gradient stenciling" also may be used to some extent. Even considering that it is more suitable for data with explicit hierarchical nature, its radial version may use the POI as the reference in order to produce better distinction of graph elements closer to the origin of a particular region.

\section{MODIFICATIONS OF FORCE-DIRECTED APPROACH TO CONTEXT-BASED DRAWING}

Obviously, traditional force-directed calculations do not take into consideration multi-attributed features of interacting element. To aid this problem, there must be a formal mechanism that enhances interaction between MFE and graph nodes in the presence of greater number of conformant attributes.

For this purpose, multi-attribution modifier coefficient $F_{m a}$ is introduced as part of scaling factor in (1) which results in (4):

$$
F_{a}^{\prime}=\left(1+F_{m a}\right) F_{a} ; F_{r}{ }^{\prime}=\left(1+F_{m a}\right) F_{r} .
$$

As it can be perceived, any $F_{m a}>0$ increases the power of mutual repulsion or attraction and a rule of thumb is that the $F_{m a}$ is proportional to the number of equal attributes $E$ in each interaction pair "MFE - affected node". The principle of calculation of $E$ is straightforward and demonstrated in part 1 of Fig. 7.
1)

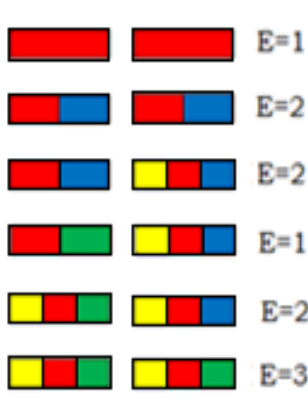

2)

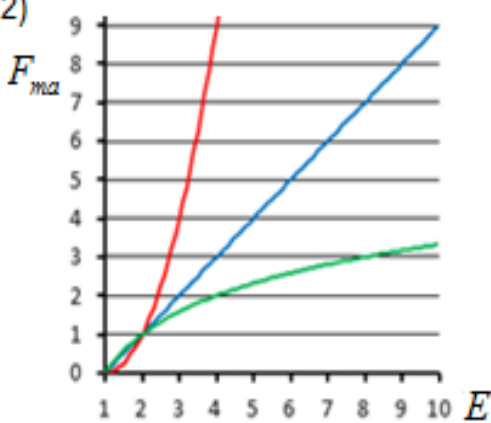

Fig. 7. Calculation of $E$ and $F_{m a}$.

Calculation of multi-attribution modifier coefficient $F_{m a}$ is based on (5):

$$
F_{m a}=F(E),
$$

where elementary function $F$ can be either a) linear; b) logarithmic or c) exponential, depending on the desired level of enforced interaction between conformant - refer to part 2 of Fig. 7 (it is important to note: +1 abscissa shift for linear and exponential functions that ensures unmodified behavior of forces if only one common attribute exists in both elements of interaction pair so that if $E=1$ then $F_{m a}=0$ ). 


\section{CASE Study}

In order to demonstrate application of the proposed approach, a case study was performed. Papers from "Scientific Journal of RTU/Applied Computer Systems" published in 2012-2014 were transformed into the multi-attributed graph (513 nodes in total including references). Nodes of the graph were attributed with the following information: Title of the Paper, Authors, Year (of publication), Keywords. References were captured in form of edges. Sample of the initial data is presented in Table 1. The respective graph is presented in $3 \mathrm{D}$ space in Fig. 8.

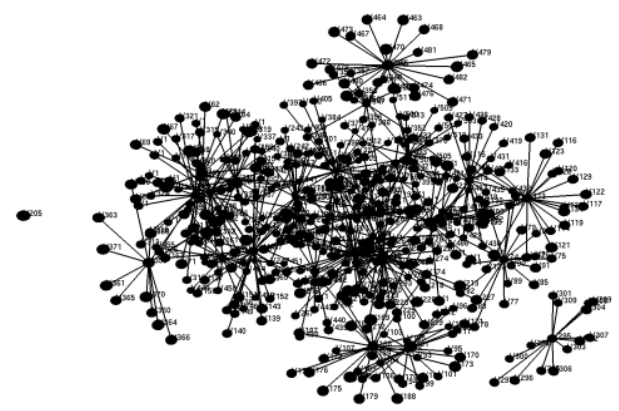

Fig. 8. Initial positioning of the graph of bibliometric network.

As the first task, we focused on retrieving from these journals those papers which were (co-)authored by [Grundspenkis] by placing appropriate MFE (marked with green color) at the center of the workspace. This yielded initial attraction and visual distinction of potentially suitable works (marked with red color) - Fig. 9.

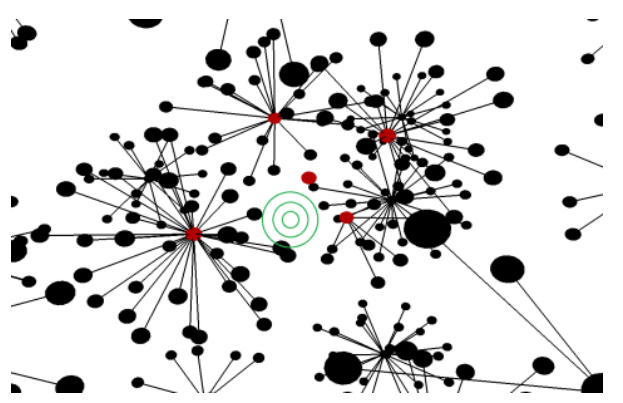

Fig. 9. The effect of application of MFE.
As the next step of processing, we narrowed the search by adding one more attribute to the MFE - keyword [intelligent agent]. This yielded stronger attraction (linear $F_{m a}$ model was used) of suitable works (marked with blue color) - Fig. 10 .

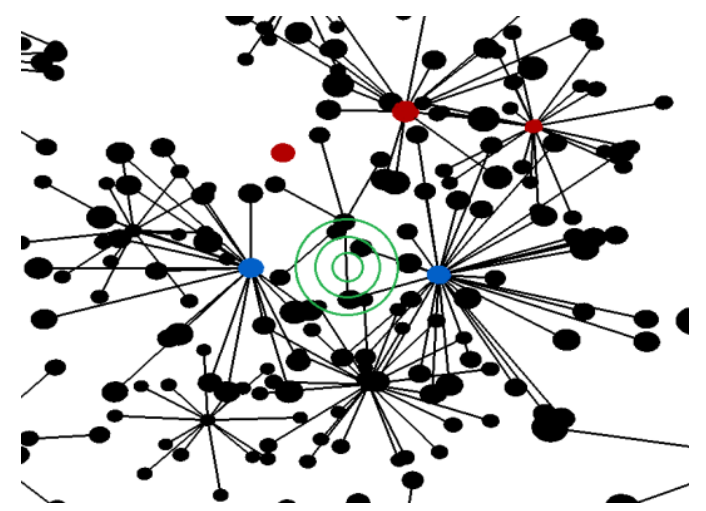

Fig. 10. The effect of adjustment of MFE.

After identification of POI, application of transparency and enabling drawing of node labels, we obtained the final result as in Fig. 11.

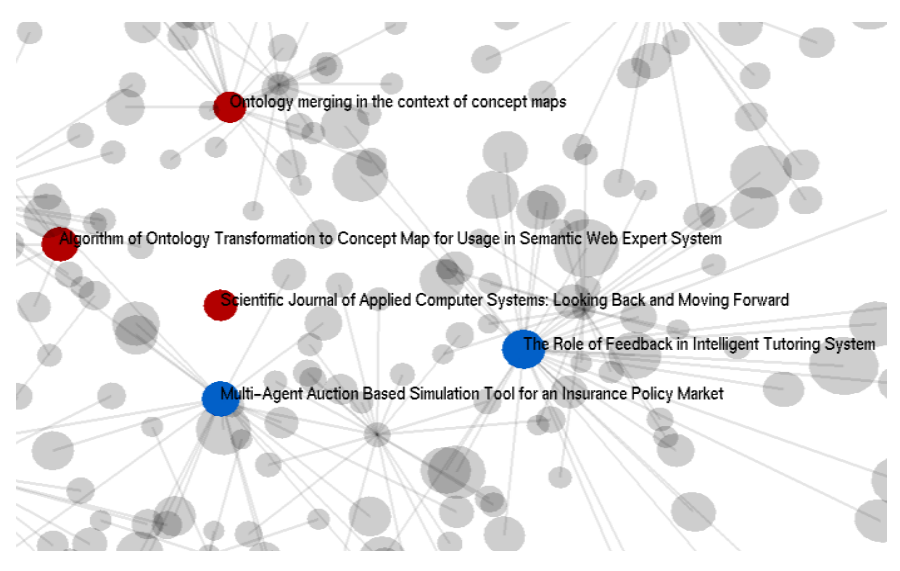

Fig. 11. Application of transparency.

This case study has demonstrated the common scenario starting from the initial unexplored dataset, continuing with gradual distinction of relevant results during goal-based search and, finally, obtaining the end result.

TABLE I

SAMPLE CONTENT OF BIBLIOMETRIC NETWORK

\begin{tabular}{|c|c|c|c|c|c|}
\hline ID & Title of the Paper & Authors & Year & Keywords & Reference count \\
\hline 1 & $\begin{array}{l}\text { Experimental Analysis of Contract Net } \\
\text { Protocol in Multi-robot Task Allocation }\end{array}$ & [Liekna]; [Lavendelis]; [Grabovskis] & 2012 & $\begin{array}{l}\text { [contract NET protocol]; } \\
\text { [multi-agent systems]; [multi- } \\
\text { robot systems]; [task allocation] }\end{array}$ & 25 \\
\hline 2 & $\begin{array}{l}\text { The Generic Map Visualization } \\
\text { Framework }\end{array}$ & [Grabovskis] & 2012 & $\begin{array}{l}\text { [design patterns]; [graphical } \\
\text { framework]; [visualization] }\end{array}$ & 13 \\
\hline 3 & $\begin{array}{l}\text { The Influence of the Map Merging } \\
\text { Order on the Resulting Global Map in } \\
\text { Multi-robot Mapping }\end{array}$ & [Andersone] & 2012 & $\begin{array}{l}\text { [map merging]; [robotic } \\
\text { mapping]; [Hough Transform]; } \\
\text { [conceptual model] }\end{array}$ & 14 \\
\hline$\ldots$ & $\ldots$ & $\ldots$ & $\ldots$ & $\ldots$ & $\ldots$ \\
\hline 513 & $\begin{array}{l}\text { Security Process Capability Model } \\
\text { Based on ISO/IEC } 15504 \text { Conformant } \\
\text { Enterprise SPICE }\end{array}$ & [Mitasiunas]; [Novickis]; [Kalpokas] & 2014 & $\begin{array}{l}\text { [Enterprise SPICE]; } \\
\text { [information security]; [process } \\
\text { capability]; [Security SPICE] }\end{array}$ & 20 \\
\hline
\end{tabular}




\section{RELATED WORK}

There are studies that are devoted to the modification of a classical force-directed approach [1]. These approaches range from general improvements of graph visualization aesthetics (such as addition of edge-to-edge repulsion for better angular resolution as in [6]) to more specific applications, e.g., [7] uses this method for better identification of preset topological configurations (called "motifs") and frequency of those in an arbitrary graph. This is done by processing nodes belonging to a motif with uniform force vector for preservation of mutual spatial positions. In [8], the author introduces to the user ability to define arbitrary boundaries of a graph and to balance its layout in real time within these boundaries, which is a useful complementary addition for combination with the MFE approach.

A number of authors concentrate on the extensive usage of visual techniques for better comprehension of graph data, e.g., [9], [10] and [11] use coloring of nodes belonging to different clusters, while [12] adds post-processing with Gaussian blur paired with transparency. The authors in [13], [14] and [15] use data visualization framework that adjusts size and label font of each node proportionally to its significance. Similar approach is utilized by the authors in [16] and [17]. These works explore a citation network graph, where the size of the node represents the number of papers written by an author, the width of the edges indicates the number of co-authored publications between two authors and the color of the nodes corresponds to different clusters of authors.

Multiple studies ([18], [19], [20], [21], [22]) rely on VOSviewer bibliometric network data visualization software that uses the concept of "density map" or "heat map" to emphasize relevant data. Colors indicate the density of relevance, ranging from blue (lowest density) to red (highest density). This principle is applied not only to separate a graph element but also to the whole workspace on a per-pixel basis to produce a continuum effect, which highly correlates with the POI/ROI concept.

Visualization of networks is performed both in twodimensional and in three-dimensional workspace. The latter is considered to have additional benefits, such as ability to distinguish data of interest visually - with heights [23], [24]. This is demonstrated in the usage of Sandia VxInsight for exploration of SCI (Science Citation Index) content [25], citation bars in [26] and usage of multiple data planes as in [27].

It is also worth mentioning that the analysis of bibliometric networks may be performed both with general-purpose visualization packages, such as in [28], [29] and dedicated software frameworks such as the solution presented in [30] for citation relations (with an emphasis on networks of co-citation relations or bibliographic coupling relations between journals/researchers/individual publications).

\section{CONCLUSION}

This research provides the analysis of adaptation of magnetic force-directed algorithms and visual techniques for context-based information extraction from multi-attributed graphs during visualization sessions. Common principles of magnetic force-directed graph drawing were outlined; magnetic force emitters, local regions of interest, anchoring of graph nodes, visual techniques and other suitable means for context-based interaction with data were described; theoretical standings for proposed changes and calculations of appropriate force coefficients were defined. Moreover, the case study and description of the current state of the art in this field were presented.

The interactive approach to extracting relevant information via MFE is a useful tool that implies not only goal-based, but also opportunity-based search and experiments with knowledge in possession of the user.

Data quality is of great importance for MFE based visualization. For example, although identification of graph nodes and edges in bibliometric network is trivial, the precision of annotating scientific works with keywords directly influences chances to obtain suitable results successfully.

Future development of this work may imply a solution to the following tasks: 1) integration of identified useful techniques (e.g., arbitrary boundaries, "density/heat map") into the presented framework and appropriate modification of these techniques for achieving mutual synergy; 2) further exploration of calculation possibilities of multi-attribution modifier coefficient $F_{m a}$ beyond linear/logarithmic/exponential and assessment of these additional models on experimenting with real datasets; 3 ) integration of all mentioned methods and techniques into a single solution that will work on "out of the box" installation basis.

\section{REFERENCES}

[1] P. Eades, "A heuristic for graph drawing" Congressus. Numerantium, vol. 42, pp. 149-160, 1984.

[2] K. Sugiyama and K. Misue, "A Simple and Unified Method for Drawing Graphs: Magnetic-Spring Algorithm" in Proceedings of the DIMACS International Workshop on Graph Drawing, GD'94, Princeton, New Jersey, USA, October 10 - 12, 1994, R. Tamassia, I. G. Tollis, Eds. Springer LNCS 894, 1995. pp. 364-375.

[3] V. Zabiniako, "Visualization of Graph Structures with Magnetic-Spring Model and Color-Coded Interaction" in Databases and Information Systems VII - Selected Papers from the Tenth International Baltic Conference, Baltic DB \& IS 2012, Vilnius, Lithuania, July 8-11, 2012, A. Caplinskas, G. Dzemyda, A. Lupeikiene, O. Vasilecas, Eds. IOS Press, 2013. pp. 106-117.

[4] V. Zabiniako, "Using Force-based Graph Layout for Clustering of Relational Data" in Advances in Databases and Information Systems: Associated Workshops and Doctoral Consortium of the 13th East European Conference ADBIS 2009: Revised Selected Papers, ADBIS 2009, Riga, Latvia, September 7-10, 2009, J. Grundspenkis, M. Kirikova, Y. Manolopoulos, L. Novickis, Eds. Berlin: SpringerVerlag Berlin Heidelberg, 2010. pp. 193-201.

[5] V. Zabiniako and P. Rusakov, "Supporting Visual Techniques for Graphs Data Analysis in Three-Dimensional Space" in Research Communications: 17th International Conference on Information and Software Technologies, IT 2011, Kaunas, Lithuania, April 27-29, 2011, R. Butleris, R. Butkiene, Eds. Kaunas: Kaunas University of Technology, 2011. pp. 75-87.

[6] C. C. Lin and H. C. Yen, "A new force-directed graph drawing method based on edge-edge repulsion" Journal of Visual Languages \& Computing, vol. 23, pp. 29-42, Feb. 2012. http://dx.doi.org/10.1016/j.jvlc.2011.12.001

[7] C. Klukas, F. Schreiber, and H. Schwoebbermeyer, "Coordinated Perspectives and Enhanced Force-Directed Layout for the Analysis of Network Motifs" in Proceedings of Asia Pacific Symposium on 
Information Visualisation, APVIS2006, Tokyo, Japan, February 1-3, 2006, K. Misue, K. Sugiyama, J. Tanaka, Eds. ACS, 2006. pp. 39-48.

[8] Y. Zhang, "Boundary Constraints in Force-Directed Graph Layout" July 2014. [Online]. Available: http://classes.soe.ucsc.edu/cmps261/ Fall13/projects/yzhang59/Final_report.pdf [Accessed: Sept. 11, 2014].

[9] A. Higgins and B. N. Smith "A Citation Based View of the Ontology Community in Philosophy" presented at WebSci '13 Web Science 2013 Paris, France, 2013.

[10] N. Shibata, Y. Kajikawa, Y. Takeda, I. Sakata, and K. Matsushima, "Early Detection of Innovations from Citation Networks" in Proceedings of the IEEE International Conference on Industrial Engineering and Engineering Management, IEEM 2009, Hong Kong, China, December 8-11, 2009, W. Kuo, M. Xie, H. Sun, R. Jiao, Eds. IEEE, 2009. pp. 54-58.

[11] L. Waltman, N. J. van Eck, and E. C. M. Noyons, "A unified approach to mapping and clustering of bibliometric networks" Journal of Informetrics, vol. 4, pp. 629-635, Oct. 2010. http://dx.doi.org/10.1016/j.joi.2010.07.002

[12] Y. Hassan-Montero, V. Guerrero-Bote, and F. Moya-Anegon, "Graphical interface of the SCImago Journal and Country Rank: Accessing an interactive approach to bibliometric information" The information professional, vol. 23, pp. 272-278, 2014.

[13] L. Gui-Feng, S. Hua-Ping, and S. Xin-Ping, "Visualizing and mapping the research on patents in information science and management science" Malaysian Journal of Library \& Information Science, vol. 19, no. 1, pp. 87-103, 2014.

[14] C. Murray, W. Ke, and K. Borner, "Mapping Scientific Disciplines and Author Expertise Based on Personal Bibliography Files" in Tenth International Conference on Information Visualization, IV 2006, London, England, July 5-6, 2006, E. Banissi, R. A. Burkhard, A. Ursyn, J. J. Zhang, M. W. McK. Bannatyne, C. Maple, A. J. Cowell, G. Y. Tian, and Ming Hou, Eds. IEEE, 2006. pp. $258-263$

[15] F. M. Leiva, M. I. Viedma, J. S. Fernandez, and A. G. Herrera, "An application of co-word analysis and bibliometric maps for detecting the most highlighting themes in the consumer behaviour research from a longitudinal perspective" Quality \& Quantity, vol. 46, no. 4, pp. 1077-1095, June. 2012.

[16] S. Grauwin and P. Jensen, "Mapping scientific institutions" Scientometrics, vol. 89, no. 3, pp. 943-954, Dec. 2011. http://dx.doi.org/10.1007/s11192-011-0482-y

[17] E. J. L. Chappin and A. Ligtvoet, "Transition and transformation: A bibliometric analysis of two scientific networks researching sociotechnical change" Renewable and Sustainable Energy Reviews, vol. 30, pp. 715-723, Feb. 2014. http://dx.doi.org/10.1016/j.rser.2013.11.013

[18] N. J. van Eck and L. Waltman "Text mining and visualization using VOSviewer" Sept. 2011. [Online]. Available: http://arxiv.org/ftp/ arxiv/papers/1 109/1109.2058.pdf [Accessed: Aug. 12, 2014].

[19] L. Leydesdorff, G. F. Khan, and L. Bornmann "The Generation of Large Networks from Web-of-Science Data" Sept. 2014. [Online]. Available http://arxiv.org/ftp/arxiv/papers/1409/1409.4898.pdf [Accessed: Sept. 22, 2014].

[20] N. J. van Eck and L. Waltman, "Software survey: VOSviewer, a computer program for bibliometric mapping" Scientometrics, vol. 84, no. 2, pp. 523-538, Aug. 2010. http://dx.doi.org/10.1007/s11192-009-0146-3

[21] F. Peset, A. Ferrer, M. Villamon, L. M. Gonzalez, J. L. Toca, and R. Aleixandre, "Scientific literature analysis of Judo in Web of Science" Archives of Budo, vol. 9, no. 2, pp. 81-91, April 2013. http://dx.doi.org/10.12659/AOB.883883

[22] E. Mostafavi and A. Bazrafshan, "Research and Collaboration Overview of Institut Pasteur International Network: A Bibliometric Approach toward Research Funding Decisions" International Journal of Health Policy and Management, vol. 2, no. 1, pp. 21-28, Jan. 2014. http://dx.doi.org/10.15171/ijhpm.2014.05

[23] V. Zabiniako and P. Rusakov, "Simultaneous Application of Graphs and Heightmaps for Enhanced Spatial Analysis" International Journal of Computer Information Systems and Industrial Management Applications, vol. 3, pp. 522-529, 2011.

[24] V. Zabiniako and P. Rusakov, "Combined Representation of Data with Graphs and Heightmaps for Visual Analysis and Spatial Decision Support" in Proceedings of the IADIS International Conferences. Computer Graphics, Visualization, Computer Vision and Image Processing 2010, IADIS 2010, Freiburg, Germany 27-29 July, 2010, Y. Xiao, R. Muffoletto, T. Amon, Eds. Germany: IADIS Press, 2010. pp. 184-192.

[25] K. W. Boyack, B. N. Wylie, and G. S. Davidson "Domain visualization using VxInsight for science and technology management" Journal of the American Society for Information Science and Technology, vol. 53, no. 9, pp. 764-774, Aug. 2002. http://dx.doi.org/10.1002/asi.10066

[26] K. Borner, C. Chen, and K. W. Boyack "Visualizing knowledge domains" Annual Review of Information Science and Technology, vol. 37, pp. 179-255, 2003. http://dx.doi.org/10.1002/aris.1440370106

[27] D. CY. Fung, S. Hong, D. Koschuenzki, F. Schreiber, and K. Xu "2.5D visualisation of overlapping biological networks" Journal of Integrative Bioinformatics, vol. 5, no. 1, pp. 1-17, 2008

[28] S. Heymann and B. L. Grand, "Visual Analysis of Complex Networks for Business Intelligence with Gephi" in Proceedings of 17th International Conference on Information Visualisation, IV 2013, London, United Kingdom, 16-18 July, 2013, E. Banissi, H. Azzag, M. W. McK. Bannatyne, S. Bertschi, F. Bouali, R. Burkhard, J. Counsell, A. Cuzzocrea, M. J. Eppler, B. Hammer, M. Lebbah, F. T. Marchese, M. Sarfraz, A. Ursyn, G. Venturini and T. G. Wyeld, Eds. IEEE, 2013. pp. 307-312.

[29] L. Bohlin, D. Edler, A. Lancichine, and M. Rosvall, "Community detection and visualization of networks with the map equation framework" Measuring scholarly impact: theory and practice, 2014 (to be published).

[30] N. J. van Eck and L. Waltman "CitNetExplorer: A new software tool for analyzing and visualizing citation networks" April. 2014. [Online]. Available: http://arxiv.org/ftp/arxiv/papers/1404/1404.5322.pdf [Accessed: Aug. 12, 2014].

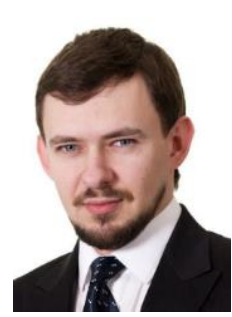

Vitaly Zabiniako was born in Riga, Latvia in 1983 Dr. sc. ing. (2012), Mg. sc. ing. (2007), Bc. sc. ing. (2005) - Riga Technical University (RTU).

IT Project Manager at IT company ABC software, previously - System Analyst (Ltd. ABC software); System Administrator (RTU, Faculty of Information Technology and Computer Science, Institute of Applied Computer Systems). Field of interests: computer science. Special interests: computer graphics, information visualization, data analysis, graph theory.

Diplomas with distinction: Bachelor of Engineering Science in Computer Control and Computer Science; Master of Engineering Science in Computer Systems.

E-mail: vitalijs.zabinako@ rtu.lv

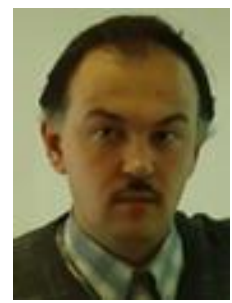

Pavel Rusakov was born in Riga, Latvia in 1972. Dr. sc. ing. (1998), Mg. sc. ing. (1995), Bc. sc. ing. (1993) - Riga Technical University (RTU).

$\mathrm{He}$ is an Associate Professor at the Institute of Applied Computer Systems, RTU. He is the Head of Laboratory, responsible for the Professional Bachelor and Professional Master studies at the Department of Applied Computer Science. Field of interests: computer science. Special interests: programming paradigms, object-oriented approach to systems development, parallel computing, Web technologies, distributed systems, computer graphics, and protection of information.

Diploma with distinction: $\mathrm{Mg}$. sc. ing.

E-mail: pavels.rusakovs@cs.rtu.lv 